\title{
Surgery for pleural mesothelioma, when it is indicated and why: arguments against surgery for malignant pleural mesothelioma
}

\author{
Gavitt A. Woodard, David M. Jablons \\ Department of Surgery, University of California, San Francisco, USA \\ Contributions: (I) Conception and design: All authors; (II) Administrative support: None; (III) Provision of study materials or patients: None; (IV) \\ Collection and assembly of data: None; (V) Data analysis and interpretation: None; (VI) Manuscript writing: All authors; (VII) Final approval of \\ manuscript: All authors. \\ Correspondence to: David M. Jablons, MD. Department of Surgery, University of California at San Francisco, 500 Parnassus Avenue, Room MUW- \\ 424, San Francisco, CA 94143-1724, USA. Email: david.jablons@ucsf.edu.
}

\begin{abstract}
Extrapleural pneumonectomy (EPP) and pleurectomy decortication (PD) are radical operations for malignant pleural mesothelioma (MPM) that remain controversial among thoracic surgeons. There is a lack of randomized evidence to support a survival benefit when major surgical resection is included in multimodality treatment regimens. Current data from retrospective single institution reviews and prospective trials such as the Surgery for Mesothelioma After Radiation Therapy (SMART) trial are limited by biased patient selection to include only the healthiest patients with most limited disease burden. This patient population predictably has relatively longer survival times than patients with inoperable advanced disease. The only randomized trial to date that has objectively evaluated the true benefit of surgical resection was the Mesothelioma and Radical Surgery (MARS) trial which actually showed shorter survival times among patients who underwent EPP compared with those treated medically. Critics of the MARS trial cite a high perioperative mortality rate for driving these results, however a similar trial has never been repeated to refute the MARS trial results. Finally, it is relevant to consider the high mortality and morbidity rates associated with major operations when recommending these interventions to MPM patients. There is a growing body of literature that identifies patients who clearly obtain no benefit from surgery including those with sarcomatoid or biphasic histology, nodal disease, elevated CRP, elevated platelets and advanced age. Surgery in MPM has risks and is of questionable benefit with outcomes data biased by patient selection of those who will have longer overall survival times regardless of treatment.
\end{abstract}

Keywords: Pleural mesothelioma; extrapleural pneumonectomy (EPP); pleurectomy decortication (PD); survival; Surgery for Mesothelioma After Radiation Therapy trial (SMART trial); Mesothelioma and Radical Surgery trial (MARS trial)

Submitted Dec 13, 2019. Accepted for publication Jan 09, 2020.

doi: $10.21037 /$ tlcr.2020.01.08

View this article at: http://dx.doi.org/10.21037/tlcr.2020.01.08

\section{Introduction}

Primum non nocere, first do no harm. Major surgical resections for malignant pleural mesothelioma (MPM) include extrapleural pneumonectomy (EPP) and pleurectomy decortication (PD). EPP involves en-bloc removal of the entire lung with visceral pleura, parietal pleura, pericardium and diaphragm for the most aggressive debulking of all possible sites of mesothelial disease. PD entails stripping the entire visceral and parietal pleura off the lung and removal of involved pericardium and diaphragm but spares the lung parenchyma in order to decrease the postoperative morbidity of a pneumonectomy and improve postoperative quality of life. Both operations are highly morbid and inevitably leave microscopic disease behind in the thorax but function as aggressive debulking procedures with the goal of obtaining a macroscopic R1 
resection. The most extreme variations of these operations involve diaphragm resection and reconstruction in order to remove all potential sites of disease, and as such this operative variation is associated with high rates of abdominal recurrence (1). Some thoracic surgeons remain convinced of the benefit of these operations as part of multi-modality treatment regimens, however others have become skeptical of their contribution to improving patient survival, especially in an era of improved chemotherapy and immunotherapy treatments.

The major criticism of current EPP and PD literature is the lack of randomized evidence and bias in patient selection which plagues all large surgical outcomes studies in MPM. The survival numbers used to promote EPP and $\mathrm{PD}$ are based on surgical resections performed in the healthiest patients, with limited disease burden and epithelial histology. The best reported survival outcomes are seen in patients who are able to complete tri-modality treatment $(2,3)$ which is a predictable conclusion that patients who survive long enough and are healthy enough to complete many months of therapy are the MPM patients who survive longer overall.

\section{Surgery for Mesothelioma After Radiation Therapy (SMART) Trial}

The best executed, most contemporary study to date to attempt to demonstrate the value of surgical resection as part of a trimodality treatment regimen is the SMART trial $(2,4)$. In the SMART trial, early-stage cT1-3N0M0 patients were treated with a short accelerated course of high-dose hypofractionated hemithoracic radiation followed by EPP and the addition of adjuvant chemotherapy for patients with pathologic N2 disease. This treatment approach is based on data that hemithoracic radiation improves local control rates in MPM but is limited by pulmonary toxicity $(1,5)$ and to administer radiation preoperatively to limit the potential for tumor spillage intraoperative during EPP. This trial took four years to accrue 25 surgical patients and it is worth noting that only $18 \%$ of 138 patients who were evaluated were ultimately eligible for the study with $51 \%$ not eligible due to advanced disease, $20 \%$ not eligible due to the presence of comorbidities and $11 \%$ refused enrollment. In this study, performed by experienced surgeons in a highvolume referral center, operative mortality was very low with $0 \% 30$-day mortality and 4\% 90-day mortality. The SMART trial initially reported a 3-year overall survival rate of $58 \%$ in patients who undergo radiation followed by EPP, however this number was based on the known survival of only 6 patients to 2 -years post-radiation therapy and only 4 patients to 3 -years (2).

Updated results using the SMART trial treatment approach in a larger cohort of 62 patients were published in 2016 with updated median survival rate of 36 months and with 36 of 62 patients alive at last follow up. The greatest benefit was again seen in patients with pure epithelial histology and ypN0 disease. This select sub-group of patients represented only 17 patients from a total of 256 who were evaluated over a 6 year period from 2008 to 2014. This group of healthy, epithelial, ypN0 patients drives the entire argument for surgery and EPP in mesothelioma, and of that group of 17 only 6 were still known to be living 3 years after the start of treatment. The authors acknowledge the risk of bias and patient selection in these studies and have noted that a randomized trial would be needed to evaluate the true benefit of this approach.

\section{Mesothelioma and Radical Surgery (MARS) trial}

To date only one prospective, randomized trial, the MARS trial, has attempted to evaluate the added benefit of a surgical resection as part of trimodality therapy. The MARS trial was conducted at 12 United Kingdom hospitals between 2005 and 2008 and registered 112 patients, to undergo induction platinum-based chemotherapy followed by randomization of 50 to EPP and postoperative hemithoracic radiation $(n=24)$ or to no EPP $(n=26)$. It is worth noting that of the original 112 patients treated with neoadjuvant chemotherapy, 33 did not progress to randomization due to disease progression, 5 did not progress due to inoperability, and 19 patients declined randomization. Of the 24 patients randomized to EPP and hemithoracic radiation only 16 had EPP completed with reasons for no completion of EPP being patient choice $(\mathrm{n}=3)$, clinical decision $(\mathrm{n}=2)$, aborted intraoperatively $(\mathrm{n}=2)$ and intraoperative death $(\mathrm{n}=1)$. Another two of the 16 EEP patients died in the initial postoperative period giving EPP a $16 \%$ perioperative mortality rate in the 19 patients in whom EPP was attempted. This high perioperative mortality rate is one of the major criticisms of the MARS trial and often cited as the reason this trial failed to show a benefit from EPP. Another death occurred outside of the 90-day postoperative window in a patient who underwent EPP and subsequently died from respiratory failure as a long-term complication of having had a pneumonectomy.

Among the 26 patients randomized to no EPP after 
neoadjuvant platinum-based chemotherapy, 10 received no further treatment while among the other 16 patients multimodality treatments were as follows: 13 were treated with additional cycles of chemotherapy, 5 received radiation treatments, 3 had non-EPP surgical management and 3 underwent EPP off trial. One of the 3 patients in the non-EPP group who underwent EPP off trial died postoperatively from multiple organ failure giving the nonsurgical arm of the trial a $33 \%$ postoperative EPP mortality rate.

Median survival in the MARS trial was 19.5 for patients randomized to no EPP and 14.4 months in the EPP group in an intention to treat analysis. One year survival rates were $52.2 \%$ in the EPP group and $73.1 \%$ in the non-EPP group with a hazard ratio (HR) of $1.90(\mathrm{P}=0.082)$ and when adjusted for prespecified prognostic risk factors the HR of EPP was $2.75(\mathrm{P}=0.016)$. It is also worth noting that none of the long-term survivors in the non-EPP group were patients that underwent EPP off trial, demonstrating that these patients had longer survival outcomes without EPP (6).

Ultimately the MARS trail failed to show any added benefit of surgery and instead demonstrated worse survival among patients who underwent EPP compared with a similar cohort of early-stage patients managed with chemotherapy alone. This trial has been legitimately criticized for its small sample size and high postoperative mortality rate among patients who underwent EPP which impacted the overall survival numbers. Nevertheless, it remains the only trial to date to randomize early-stage surgical candidates to non-operative management and importantly demonstrated comparable survival with chemotherapy and radiation treatments alone. The median survival time of 19.5 months from randomization does not include an additional median 3.6 months from the time of registration to randomization, which puts the total median survival times at 23.1 months from the time of enrollment for patients in the non-EPP arm of the trial. This number is comparable to, if not better than, most published single center survival rates following EPP and PD from major mesothelioma treatment centers.

\section{Retrospective single institution EPP and PD outcomes}

Beyond the SMART trial, the remaining literature used to justify aggressive surgical resections such as EEP or extended PD relies heavily on single-institution series of early-stage, limited disease burden, epithelioid histology patients who are hand-picked for surgical resection leading to an inherent bias when reporting long-term survival outcomes in this group of patients post-operatively. Despite the selection of patients who based on baseline clinical characteristics are expected to have longer survival times, median survival following a major debulking surgery is routinely cited as 14-18 months following either EPP or $\mathrm{PD}$, essentially the same as non-operative patients (7-11) particularly when compared to the early stage patients from the MARS trial which had a median survival of 19.5 months when treated non-operatively (6).

One of the more recent reviews to summarize all of the evidence on survival outcomes following EPP and PD combined global published outcomes from a total of 5,113 surgically treated MPM patients through mostly retrospective reviews. In these large published series EEP had a median overall survival of 4.7 to 23 months and PD had a median overall survival of 10 to 32 months leading the authors to summarize that operative mortality and morbidity is higher with EPP and that overall survival is similar or lower following EPP compared with PD (12).

Retrospective studies published from major referral centers in the United States that are often cited for survival outcomes include a 945 patient experience from Memorial Sloan Kettering from 1990 to 2005 in which 208 patients underwent EPP and 176 patients underwent PD. Patients operated on at this high volume referral center had a median survival of 14.3 months for patients who underwent EPP and 15.8 months for patients who underwent PD. This compared with 12.7 months median survival in patients who had an exploratory thoracotomy only without a major resection and 10.2 months in patients who were managed totally non-operatively (9). These survival outcomes for diagnostic VATS or palliative decortication are similar to 12 month median survival times cited in several other studies of unselected, advanced stage patients with epithelial, biphasic, and sarcomatoid histology $(13,14)$.

The 24-year experience from 1988 to 2011 at Brigham and Women's Hospital included 529 patients with epithelial histology MPM who underwent EPP as part of multimodality treatments. These patients had a median survival rate of 18 months with 1-, 3-, 5-, and 10-year survival rates of $67 \%, 28 \%, 14 \%$, and $4 \%$, respectively (7). While these retrospective studies are often used to advocate for the survival advantage of surgery, patients who underwent EEP or PD in these studies were those with the most limited disease at the time of diagnosis. Any survival differences between surgically managed patients and those 
treated with only chemotherapy and/or radiation is biased by early stage disease in surgically managed patients. A randomized trial between surgical and non-surgical management would be necessary to support the claim that surgery improves long-term survival in mesothelioma in early stage patients.

\section{Characteristics that predict poor survival after surgery}

Trends have emerged regarding patients with better and worse outcomes following EPP and PD which point to predictors of advanced disease and poor prognosis that identify patient populations which certainly do not benefit from surgery. These predictors of poor survival outcome postoperatively include: biphasic or sarcomatoid histology, pN1 or N2 disease (7), elevated CRP, elevated platelets and patients with these characteristics are known to have shorter overall survival times and no benefit from surgical resection. Countless studies have demonstrated poor survival among patients with sarcomatoid or biphasic histology, with one study using the Surveillance, Epidemiology, and End Results (SEER) database on outcomes from patients who underwent all types of surgical resection demonstrating median overall survival of 19 months among patients with epithelial histology, 12 months among patients with biphasic histology, and 4 months among pure sarcomatoid histology patients (15). In the large $529 \mathrm{EPP}$ patient study older age and male gender were associated with worse survival outcomes with age over 59 years having a median survival of 16 months versus 20 months in patients younger than $59(\mathrm{P}=0.0009)$. Women had median survival of 28 months compared with 17 months in men $(\mathrm{P}=0.005)$.

Preoperative laboratory values of CRP, platelet count, leukocyte count and LDH can also be predictive of longterm survival outcomes after major surgical resection (13). One retrospective multicenter study from Austria on the acute phase reactive protein CRP found that elevated levels of preoperative CRP were associated with very poor survival in patients who underwent surgery as part of multi-modality therapy for MPM. Median survival among patients who underwent surgery with normal CRP levels $(<1.0 \mathrm{mg} / \mathrm{dL})$ was 35.9 vs. 9.7 months median survival of patients with CRP levels above $1.0 \mathrm{mg} / \mathrm{dL}$ (16). These serum biomarkers of poor prognosis are not independent of other risk factors and likely reflect systemic inflammation associated with more advanced stage mesothelioma, they nevertheless might be used to identify patients who will have poor outcomes postoperatively. As patients with these characteristics obtain less benefit from surgery, it is not worth the morbidity and potential mortality of surgery to subject them to major operations which may ultimately delay other treatments such as radiation or chemotherapy.

On the other end of the spectrum are patients with predictors of slow disease progression and long life expectancy regardless of treatment. Patients with genetic predisposition to MPM identified by family history of MPM or early age at diagnosis of MPM have significantly longer median survival times than those with sporadic mesothelioma. MPM patients with family history of MPM and a germline BRCA1-associated protein (BAP) mutation have median survival times of 5 years whereas MPM patients with a family history of MPM and wildtype BAP have median survival times of 9 years (17). Given the incredible survival in this group, reflecting a different biologically determined pace of disease progression, the benefits of aggressive and morbid surgical treatments must be questioned.

\section{Mortality and morbidity of surgery}

In addition to lack of a proven benefit, there is also a significant risk of mortality and morbidity following a major surgical resection such as EPP or extended PD that is often overlooked. Even at the most experienced, high-volume centers 30-day or in-hospital mortality following EPP is reported at $5-7 \%(7-11)$ with postoperative mortality rates at the very best high-volume mesothelioma programs more than doubling to $11 \%$ when patients are followed up to 90 days postoperatively (18). For the patients that do survive surgery the majority of literature cites complication rates as high as $45 \%$ (18) and these studies also do not address the pain and suffering that patients endure in order to recover from a large thoracotomy, rib shingling or removal, with or without pneumonectomy. Patients who undergo pneumonectomy have diminished respiratory function, may forever require supplemental oxygen, and have diminished quality of life. For these reasons, many thoracic surgeons have chosen to no longer perform EPP and instead favor extended PD for mesothelioma. Survival outcomes improved from 15.6 months to 19.6 months in a center with the same surgeons and patient population when the practice of EPP was abandoned following publication of the MARS trial in 2011 (19). Several meta-analyses have all favored PD over EPP due to the higher mortality following EPP without a survival benefit over PD $(8,10,20)$. 


\section{Conclusions}

There is a lack of randomized trial evidence to support any survival benefit when MPM patients undergo a major surgical resection such as EPP or PD. All of the current literature that advocates for surgical resection is biased by patient selection for the earliest stage, healthiest patients. The only randomized trial to date, the MARS trial, showed no benefit to EPP over non-surgical treatments and many thoracic surgeons believe that major surgical resections in mesothelioma do not improve survival and cannot be justified except in rare instances. However, there are many surgeons who continue to perform EPP or PDs routinely in the absence of data and so the role of surgery in mesothelioma remains controversial. Arguments in support of surgical resection for mesothelioma are concisely explained in a recent mesothelioma review by Carbone et al. that explains both sides of this debate (21). We believe that a randomized controlled trail in which nonsurgical multimodality management is compared to aggressive operative approaches is needed to justify continuing the practice of EPP and PD for mesothelioma.

In the meantime other novel therapies are showing promise, making major surgical resections an even more questionable treatment decision. Studies of immunotherapy in mesothelioma are ongoing. As part of KEYNOTE-028 25 patients with anti-programmed cell death receptor ligand 1 (PD-L1) positive MPM were treated with the PD-1 antibody pembrolizumab and $20 \%$ of these patients had a partial response and $52 \%$ had stable disease with a durable response time of 12 months (22). Chimeric antigen receptor (CAR)-modified autologous $\mathrm{T}$ cells directed against mesothelin is another innovative approach with growing evidence. After much preclinical work (23), a phase I study was just completed in 15 patients with chemotherapy refractory MPM, ovarian carcinoma, and pancreatic ductal adenocarcinoma. Patients were treated with a single infusion of CART-meso cells engineered by lentiviral transduction with limited toxicity and 11 of 15 experienced stable disease in response to a single treatment with CART-meso DNA was detected in $70 \%$ of subsequent tumor biopsies (24). With novel and improved chemo and immunotherapies in the pipeline, surgical approaches and outcomes for MPM remain unchanged and their questionable benefit will ultimately be eclipsed by other systemic treatments.

\section{Acknowledgments}

Funding: None.

\section{Footnote}

Conflicts of Interest: The authors have no conflicts of interest to declare.

Ethical Statement: The authors are accountable for all aspects of the work in ensuring that questions related to the accuracy or integrity of any part of the work are appropriately investigated and resolved.

Open Access Statement: This is an Open Access article distributed in accordance with the Creative Commons Attribution-NonCommercial-NoDerivs 4.0 International License (CC BY-NC-ND 4.0), which permits the noncommercial replication and distribution of the article with the strict proviso that no changes or edits are made and the original work is properly cited (including links to both the formal publication through the relevant DOI and the license). See: https://creativecommons.org/licenses/by-ncnd/4.0/.

\section{References}

1. Gomez DR, Hong DS, Allen PK, et al. Patterns of failure, toxicity, and survival after extrapleural pneumonectomy and hemithoracic intensity-modulated radiation therapy for malignant pleural mesothelioma. J Thorac Oncol 2013;8:238-45.

2. Cho BC, Feld R, Leighl N, et al. A feasibility study evaluating Surgery for Mesothelioma After Radiation Therapy: the "SMART" approach for resectable malignant pleural mesothelioma. J Thorac Oncol 2014;9:397-402.

3. de Perrot M, Feld R, Cho BC, et al. Trimodality therapy with induction chemotherapy followed by extrapleural pneumonectomy and adjuvant high-dose hemithoracic radiation for malignant pleural mesothelioma. J Clin Oncol 2009;27:1413-8.

4. de Perrot M, Feld R, Leighl NB, et al. Accelerated hemithoracic radiation followed by extrapleural pneumonectomy for malignant pleural mesothelioma. J Thorac Cardiovasc Surg 2016;151:468-73.

5. de Perrot M, Uy K, Anraku M, et al. Impact of lymph node metastasis on outcome after extrapleural pneumonectomy for malignant pleural mesothelioma. J Thorac Cardiovasc Surg 2007;133:111-6.

6. Treasure T, Lang-Lazdunski L, Waller D, et al. Extrapleural pneumonectomy versus no extra-pleural pneumonectomy for patients with malignant pleural 
mesothelioma: clinical outcomes of the Mesothelioma and Radical Surgery (MARS) randomised feasibility study. Lancet Oncol 2011;12:763-72.

7. Sugarbaker DJ, Richards WG, Bueno R. Extrapleural pneumonectomy in the treatment of epithelioid malignant pleural mesothelioma: novel prognostic implications of combined N1 and N2 nodal involvement based on experience in 529 patients. Ann Surg 2014;260:577-80; discussion 580-2.

8. Flores RM, Pass HI, Seshan VE, et al. Extrapleural pneumonectomy versus pleurectomy/decortication in the surgical management of malignant pleural mesothelioma: results in 663 patients. J Thorac Cardiovasc Surg 2008;135:620-6, 626.e1-3.

9. Flores RM, Zakowski M, Venkatraman E, et al. Prognostic factors in the treatment of malignant pleural mesothelioma at a large tertiary referral center. J Thorac Oncol 2007;2:957-65.

10. Cao C, Tian D, Park J, et al. A systematic review and meta-analysis of surgical treatments for malignant pleural mesothelioma. Lung Cancer 2014;83:240-5.

11. Schipper PH, Nichols FC, Thomse KM, et al. Malignant pleural mesothelioma: surgical management in 285 patients. Ann Thorac Surg 2008;85:257-64; discussion 264.

12. Sayan M, Eren MF, Gupta A, et al. Current treatment strategies in malignant pleural mesothelioma with a treatment algorithm. Adv Respir Med 2019;87:289-97.

13. Rena O, Boldorini R, Papalia E, et al. Persistent lung expansion after pleural talc poudrage in non-surgically resected malignant pleural mesothelioma. Ann Thorac Surg 2015;99:1177-83.

14. Baud M, Strano S, Dechartres A, et al. Outcome and prognostic factors of pleural mesothelioma after surgical diagnosis and/or pleurodesis. J Thorac Cardiovasc Surg 2013;145:1305-11.

15. Meyerhoff RR, Yang CF, Speicher PJ, et al. Impact of mesothelioma histologic subtype on outcomes in the Surveillance, Epidemiology, and End Results database. J

Cite this article as: Woodard GA, Jablons DM. Surgery for pleural mesothelioma, when it is indicated and why: arguments against surgery for malignant pleural mesothelioma. Transl Lung Cancer Res 2020;9(Suppl 1):S86-S91. doi: 10.21037/ tlcr.2020.01.08
Surg Res 2015;196:23-32.

16. Ghanim B, Hoda MA, Winter MP, et al. Pretreatment serum C-reactive protein levels predict benefit from multimodality treatment including radical surgery in malignant pleural mesothelioma: a retrospective multicenter analysis. Ann Surg 2012;256:357-62.

17. Pastorino S, Yoshikawa Y, Pass HI, et al. A Subset of Mesotheliomas With Improved Survival Occurring in Carriers of BAP1 and Other Germline Mutations. J Clin Oncol 2018:JCO2018790352.

18. McMillan RR, Berger A, Sima CS, et al. Thirty-day mortality underestimates the risk of early death after major resections for thoracic malignancies. Ann Thorac Surg 2014;98:1769-74; discussion 1774-5.

19. Batirel HF, Metintas M, Caglar HB, et al. Adoption of pleurectomy and decortication for malignant mesothelioma leads to similar survival as extrapleural pneumonectomy. J Thorac Cardiovasc Surg 2016;151:478-84.

20. Taioli E, Wolf AS, Flores RM. Meta-analysis of survival after pleurectomy decortication versus extrapleural pneumonectomy in mesothelioma. Ann Thorac Surg 2015;99:472-80.

21. Carbone M, Adusumilli PS, Alexander HR, Jr., et al. Mesothelioma: Scientific clues for prevention, diagnosis, and therapy. CA Cancer J Clin 2019;69:402-29.

22. Alley EW, Lopez J, Santoro A, et al. Clinical safety and activity of pembrolizumab in patients with malignant pleural mesothelioma (KEYNOTE-028): preliminary results from a non-randomised, open-label, phase $1 \mathrm{~b}$ trial. Lancet Oncol 2017;18:623-30.

23. Thayaparan T, Petrovic RM, Achkova DY, et al. CAR T-cell immunotherapy of MET-expressing malignant mesothelioma. Oncoimmunology 2017;6:e1363137.

24. Haas AR, Tanyi JL, O'Hara MH, et al. Phase I Study of Lentiviral-Transduced Chimeric Antigen ReceptorModified T Cells Recognizing Mesothelin in Advanced Solid Cancers. Mol Ther 2019;27:1919-29. 